\title{
Arab state pours oil profits into science
}

How would you spend the profits from an oil well? That question was on the mind of about 200 Arab scientists who gathered in Doha, Qatar, late last month.

The country's head of state, Emir Hammad bin Khalifa Al-Thani, has devoted a chunk of Qatar's fossil-fuel profits to research, and the region's expatriate scientists were brought together to advise how best to invest it. Get it right, said attendees, and the money could help create an internationally respected science base for the Arab world.

"If it succeeds, it will change the whole region," says Hilal Lashuel, a Yemeni neuroscientist at the Swiss Federal Institute of Technology (ETH) in Lausanne. "Arabs will compete for the first time."

Qatar is already gaining scientists' attention in part because the country has recently transformed its university system. The tiny Gulf state, which has a population of less than 900,000 , supports Western standards of living thanks to its substantial oil and gas fields. But those reserves are limited.

Hence Qatar's decision to jump on the knowledge-economy bandwagon and create Education City. The 2,500-acre campus on the outskirts of Doha hosts undergraduate teaching branches of several well-known US universities, such as Texas A\&M. By replicating Western academic culture in the Gulf, the country's leaders hope to eventually attract 2,000 students annually to what could become the region's premier teaching facility. Several hundred students are already enrolled.

Education City has been bankrolled by the Qatar Foundation for Education, Science and Community Development. This is funded by an endowment from Al-Thani that officials say runs to billions of dollars. With this education centre up and running, the foundation is now turning its attention to applied research and the income-generating technologies that flow from it.

The emir has set aside profits from one of the country's oil wells for the purpose. Combined with a contribution from the foundation's endowment, Qatar will have a dedicated research fund worth hundreds of millions of dollars a year.

\section{"Arabs will compete for the first time."} international terms, but Arab scientists say that the other components of Qatar's research vision show that the country means business. Foreign labs are being enticed to a science park on the Education City site, for example, by offers of state-of-the-art research

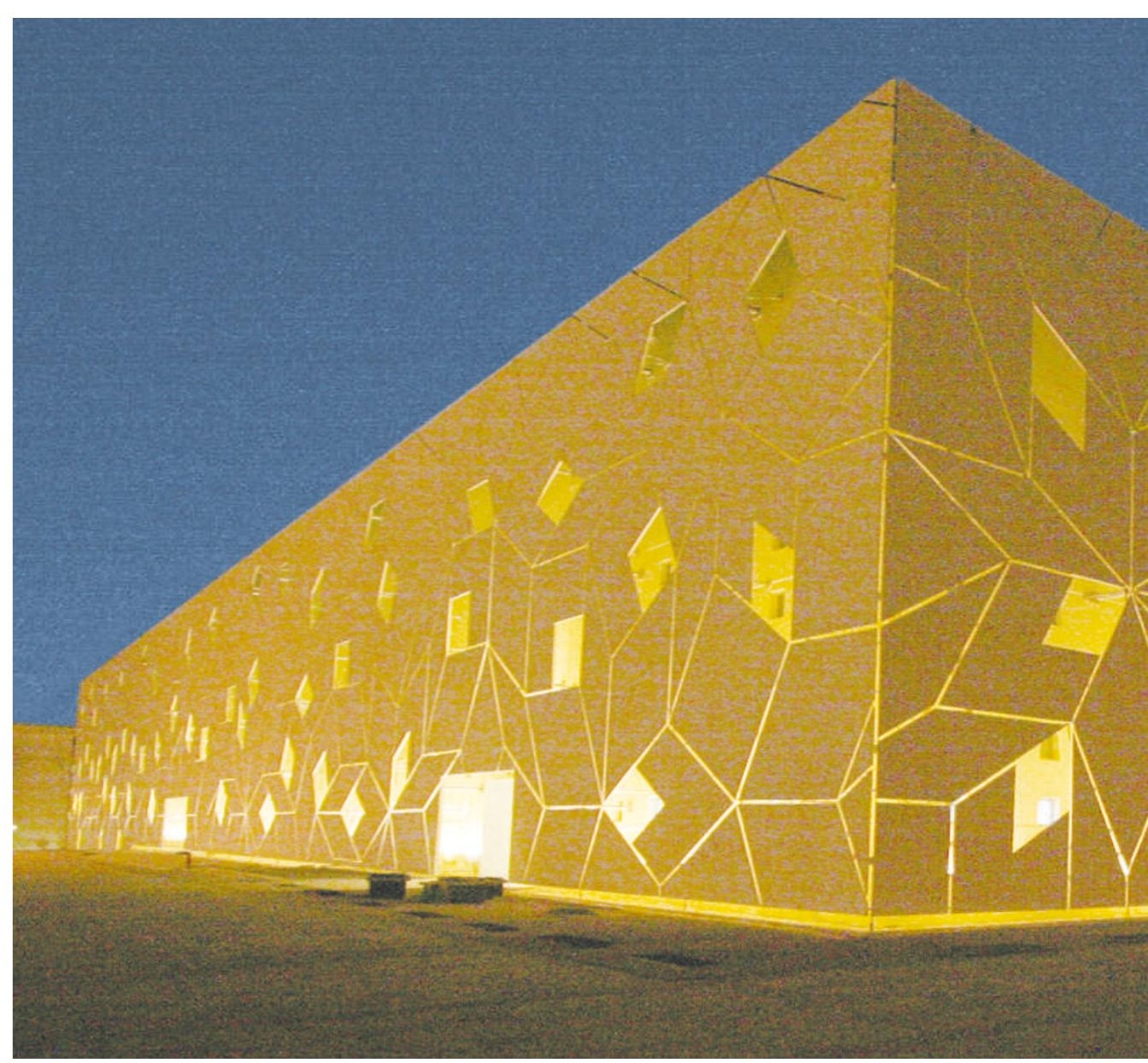

Qatar's recently launched teaching centre, Education City, is helping to attract researchers from overseas.

facilities and liberal intellectual-property agreements. All the labs have to do is provide the staff. It is a deal that has attracted interest from research institutions such as Imperial College London and Tokyo University.

\section{Tempting talent}

Tidu Maini, Imperial's pro-rector, says his university is working with the foundation on plans for a diabetes genome centre. The Gulf region has one of the highest rates of diabetes in the world, making it the ideal place to probe the genetics of the disease. Although plans are at an early stage, Maini says research groups could be set up this year at Imperial and the Hamad General Hospital in Qatar. The researchers would eventually move to the new genome centre, which Maini says could open in about three years. He would like every newborn child in Qatar, and their parents, to be genotyped in order to build up a database that could be probed for diabetes studies.

Qatar is importing Western practices as well as personnel. The country's research fund, for example, will be administered by independent peer-review panels. These will be composed of researchers from home and abroad, and modelled on processes followed by organizations such as the US National Institutes of Health. Fathy Saoud, a parasitologist who sits on the Qatar foundation's board of directors, says that grant applications for biomedical, environmental and computing projects will be considered in about a year's time.

At last month's conference, held from 24 to 26 April, expatriate Arab researchers talked about how the Qatar project could boost science investment across the region. Researchers say that if the initiative takes off, it could force neighbouring countries to launch similar 
